\title{
Conformations and charge transport characteristics of biphenyldithiol self-assembled-monolayer molecular electronic devices: A multiscale computational study
}

\author{
Yong-Hoon $\mathrm{Kim}^{\mathrm{a}}$ \\ Materials and Process Simulation Center, California Institute of Technology, Pasadena, \\ California 91125-7400 and Korea Institute for Advanced Study, 207-43 Cheongnyangni 2-dong, \\ Dongdaemun-gu, Seoul, 130-722 Korea
}

Seung Soon Jang and William A. Goddard III)

Materials and Process Simulation Center, California Institute of Technology, Pasadena, California 91125-7400

(Received 26 January 2005; accepted 25 April 2005; published online 27 June 2005)

\begin{abstract}
We report a computational study of conformations and charge transport characteristics of biphenyldithiol (BPDT) monolayers in the $(\sqrt{3} \times \sqrt{3}) R 30^{\circ}$ packing ratio sandwiched between $\mathrm{Au}(111)$ electrodes. From force-field molecular-dynamics and annealing simulations of BPDT self-assembled monolayers (SAMs) with up to 100 molecules on a $\mathrm{Au}(111)$ substrate, we identify an energetically favorable herringbone-type SAM packing configuration and a less-stable parallel packing configuration. Both SAMs are described by the $(2 \sqrt{3} \times \sqrt{3}) R 30^{\circ}$ unit cell including two molecules. With subsequent density-functional theory calculations of one unit cell of the (i) herringbone SAM with the molecular tilt angle $\theta \approx 15^{\circ}$, (ii) herringbone SAM with $\theta \approx 30^{\circ}$, and (iii) parallel SAM with $\theta \approx 30^{\circ}$, we confirm that the herringbone packing configuration is more stable than the parallel one but find that the energy variation with respect to the molecule tilting within the herringbone packing is very small. Next, by capping these SAMs with the top $\mathrm{Au}(111)$ electrode, we prepare three molecular electronic device models and calculate their coherent charge transport properties within the matrix Green's function approach. Current-voltage $(I-V)$ curves are then obtained via the Landauer-Büttiker formula. We find that at low-bias voltages $(|V| \lesssim 0.2 \mathrm{~V})$ the $I-V$ characteristics of models (ii) and (iii) are similar and the current in model (i) is smaller than that in (ii) and (iii). On the other hand, at higher-bias voltages $(|V| \geqslant 0.5 \mathrm{~V})$, the $I-V$ characteristics of the three models show noticeable differences due to different phenyl band structures. We thus conclude that the BPDT SAM $I-V$ characteristics in the low-bias voltage region are mainly determined by the $\mathrm{Si}-\mathrm{Au}$ interaction within the individual molecule-electrode contact, while both intramolecular conformation and intermolecular interaction can affect the BPDT SAM $I-V$ characteristics in the high-bias voltage region. (C) 2005 American Institute of Physics.
\end{abstract}

[DOI: $10.1063 / 1.1937391]$

\section{INTRODUCTION}

The recent developments in techniques of solid-state device fabrication, chemical synthesis, and atomic resolution characterization tools make it feasible now to construct molecular electronic devices and study their charge transport properties. ${ }^{1-3}$ However, controlling the synthesis and measuring the characteristics of the molecular-scale electronic devices is still a challenging task due to the high complexity, low observability, and limited controllability of the experiments. Rapid progress in the field demands the development of theoretical methods to model the molecular electronic devices and to characterize their performance in advance of experiment. In this work, we present a computational approach addressing the charge transport properties of molecu-

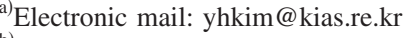

b) Author to whom correspondence should be addressed. Electronic mail: wag@wag.caltech.edu
}

lar electronic devices based on self-assembled monolayers (SAMs) in correlation with molecular conformations including the SAM packing.

An important strategy in fabricating molecular electronic devices is preparing SAM structures on an electrode and attaching a second electrode in such a way as to form wires, diodes, switches, etc. ${ }^{4}$ The flexible nature of individual organic molecules and their rather unstable attachment to the electrodes can make the control of devices based on single molecules very difficult. On the other hand, intermolecular interactions within the SAM can possibly provide welldefined molecular structures and correspondingly a more robust device control mechanism.

We will consider here molecular electronic devices made by sandwiching biphenyldithiol (BPDT) SAMs between $\mathrm{Au}(111)$ electrodes (Fig. 1). Phenyl and phenyleneethynylene-based molecules thiol bridged to $\mathrm{Au}$ electrodes have been extensively employed in recent experiments. ${ }^{5-11}$ Theoretical studies of current-voltage $(I-V)$ characteristics 
have been also reported with different methodologies at varying levels of computational complexity. ${ }^{8,12-20}$ However, these works were mostly limited to single-molecule models in their ideal geometry. Since charge transport properties at the molecular scale would depend sensitively on the intramolecular geometry and the contact configuration, we believe that it is crucial to obtain realistic conformations of the molecule-electrode composite systems before considering their $I-V$ characteristics. We address the complex problem of the structure $-I-V$ characteristics correlation in molecular electronic devices by employing a multiscale computational approach combining density-functional theory (DFT), forcefield $(\mathrm{FF})$, and matrix Green's-function (MGF) methods within the two-dimensional periodic boundary condition. ${ }^{21}$ Carrying out large-scale molecular dynamics and energy minimizations with FFs based on DFT calculations of simple model systems, we first generate plausible BPDT SAM structures. Device models are prepared by covering the SAMs with the top electrode, and their $I-V$ characteristics are calculated using the DFT-MGF method. Especially, we focus on how the $I-V$ characteristics correlate with the molecular configuration and the SAM packing. This approach has been successfully applied to the study of the structures and $I-V$ characteristics of the [2]catenane molecular electronic switches. ${ }^{21}$

The organization of the paper is as follows. In Sec. II, we will report BTDT SAM structures obtained by the combination of large-scale FF molecular-dynamics simulations plus geometry optimizations and subsequent DFT optimizations with smaller unit cells. Within the ideal $(\sqrt{3}$ $\times \sqrt{3}) R 30^{\circ}$ packing ratio which corresponds well to the BPDT crystal structure and a plausible SAM packing density, we find two competing packing configurations. In addition to the packing conformation, molecular tilting with respect to the electrode surface is another variable we consider. Geometries and energetics of SAMs with different packing conformations and molecular tilt angles will be compared within FFs. Three SAM configurations will be particularly selected for more accurate DFT energy minimizations. In Sec. III, we will consider the BPDT SAM device models prepared by covering the three SAMs with the top electrode. Their $I-V$ characteristics are computed and analyzed using the MGF formalism. We will focus on the effect of molecule tilting and SAM packing configuration on the device $I-V$ characteristics. Section IV will summarize the current work.

\section{MONOLAYER STRUCTURES FROM FORCE-FIELD AND DENSITY-FUNCTIONAL CALCULATIONS}

The first step of fabricating a molecular-scale electronic device of interest here is assembling a SAM film on a metal substrate, which we consider to be the (111) surface of Au. It is well known that alkanethiols adsorb on $\mathrm{Au}(111)$ in $c(4 \sqrt{3} \times 2 \sqrt{3}) R 30^{\circ}$ superstructures with the molecular tilt angle of $30^{\circ}$. However, conformation and packing in SAMs based on phenyl thiolates are not yet fully understood. To determine the structure of the BPDT SAM on Au(111) [Fig. 1(a)], we first employed large-scale FF molecular-dynamics and geometry optimizations to search candidate SAM con-

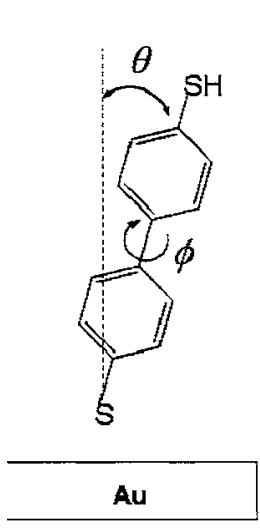

(a)

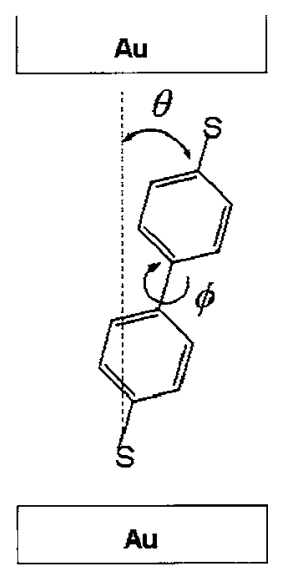

(b)
FIG. 1. BPDT molecule models employed in (a) the self-assembly process and (b) $I-V$ characteristics calculations. Relevant geometrical variables are tilt angle $\theta$ and torsion angle $\phi$.

figurations. For this purpose, we extended the Dreiding FF (Ref. 22) to include $\mathrm{Au}-\mathrm{Au}$ and BPDT-Au interactions through nonbonded potential energy in the exponential-6type van der Waals form. ${ }^{23}$ The resulting FF predicts the binding energy of $1.17 \mathrm{eV}$ for the chemisorption of alkanethiol molecule on a $\mathrm{Au}(111)$ surface, in good agreement with the experimental value of $1.31 \mathrm{eV}$ for the homolytic reaction of alkylthiol onto a Au surface. ${ }^{24}$ Details of our FFs are reported elsewhere. ${ }^{23}$ The atomic charges required for the evaluation of the electrostatic energy were calculated using the charge equilibration method. ${ }^{25}$

To determine the structure of the SAMs, we first constructed a slab with six layers of $\mathrm{Au}$ atoms by repeating the $(\sqrt{3} \times \sqrt{3}) R 30^{\circ}$ unit cell with the experimental lattice constant from $4 \times 4$ to $10 \times 10$ times. BPDT molecules were then placed in random configurations at the one molecule per $(\sqrt{3} \times \sqrt{3}) R 30^{\circ}$ cell density (thus including from 16 to 100 BPDT molecules). This packing density, which leads to the densest packing and accordingly the strongest intermolecular interactions for the thiol-based SAMs, not only corresponds well to the biphenyl crystal structure but was also observed in the experiments. ${ }^{26,27}$ However, as will be detailed later, SAMs made of biphenylthiol molecules on Au(111) are experimentally described as not very well ordered. So, our perfectly ordered packing models should be considered as an idealized situation.

The Au(111)-BPDT slab was separated from its periodic images by $10 \AA$ or more of vacuum space along the surface normal direction within the periodic boundary condition to minimize artificial interactions of molecules with image metal atoms in the repeating unit cells. Fixing the bottom one layer of $\mathrm{Au}$ atoms, we performed canonical ensemble (NVT) molecular-dynamics simulations at $300 \mathrm{~K}$ with the NoseHoover thermostat ${ }^{28}$ for 100 ps with a time step of $1 \mathrm{fs}$ to equilibrate the system out of the local energy minima, and then optimized the structure to minimize the energy. The resulting SAM on $\mathrm{Au}(111)$ shows an ordered herringbone packing of BPDT molecules corresponding to a $(2 \sqrt{3}$ 

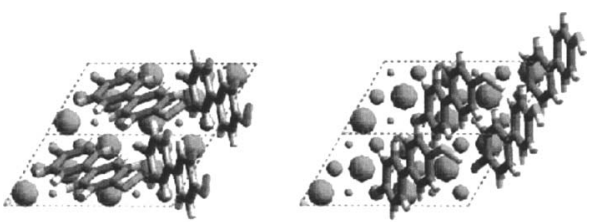

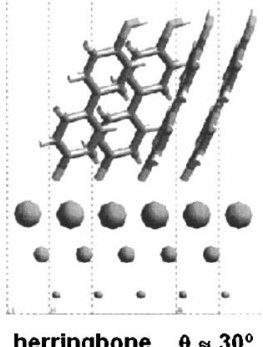

(a)

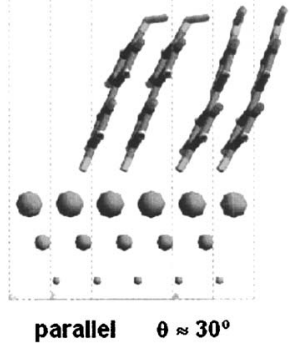

(b)
FIG. 2. Top and side views of $1 \times 2(2 \sqrt{3} \times \sqrt{3}) R 30^{\circ}$ cell BPDT SAM on the $\mathrm{Au}(111)$ electrode in the (a) herringbone $\theta \approx 30^{\circ}$ and (b) parallel $\theta \approx 30^{\circ}$ configurations. Color codes: $\mathrm{C}$ (grey), $\mathrm{H}$ (white), $\mathrm{S}$ (yellow), and $\mathrm{Au}$ (orange).

$\times \sqrt{3}) R 30^{\circ}$ unit-cell superstructure anchored by $\mathrm{S}$ atoms approximately at the face-centered-cubic (fcc) hollow sites of $(\sqrt{3} \times \sqrt{3}) R 30^{\circ}$ as shown in Fig. 2(a).

In addition to the herringbone packing, within the $(2 \sqrt{3}$ $\times \sqrt{3}) R 30^{\circ}$ unit-cell configuration, we also obtained a parallel packing structure of Fig. 2(b) by applying local constraints. In this packing mode, while one $\mathrm{S}$ atom is located at the fcc site, the other $\mathrm{S}$ atom is at the hexagonal-closepacked (hcp) site. This configuration is energetically higher than the herringbone one by $\sim 0.15 \mathrm{eV}$ per molecule within our FFs. Two phenyl rings are coplanar, $\phi=0^{\circ}$, in both the herringbone and parallel SAM structures due to intermolecular interactions, in contrast to the twisted geometry in the isolated molecular configuration [e.g., in the isolated BPDT $\left(\mathrm{SH}-\mathrm{C}_{6} \mathrm{H}_{4}-\mathrm{C}_{6} \mathrm{H}_{4}-\mathrm{SH}\right)$ molecule, the twisting angle between two phenyl rings is $\phi=36.9^{\circ}$ from DFT with B3LYP $/ 6-31 G^{* *}$ and $38.7^{\circ}$ from our FF]. Molecular tilt angles defined via $\mathrm{S}-\mathrm{S}$ axes with respect to the electrode surface normal direction are $\theta=26.2^{\circ}$ and $\theta=28.0^{\circ}$ within the herringbone and parallel packing configurations, respectively. The geometries and energetics of the two structures are summarized in Tables I and II. To check the relaxation effect of the Au surface atoms, we also considered the case where all the $\mathrm{Au}$ atoms are fixed. Comparing the two cases, we find that the Au relaxation has negligible effect on the relative $\mathrm{Au}-\mathrm{BPDT}$ molecule conformations.

We now consider experiments of biphenyl-based SAMs. Studies employing monolayers of 4-mercaptobiphenyl $\left(\mathrm{C}_{6} \mathrm{H}_{5}-\mathrm{C}_{6} \mathrm{H}_{4}-\mathrm{SH}\right) \quad$ (Ref. 29) and 4-methyl4'-mercaptobiphenyl $\left(\mathrm{CH}_{3}-\mathrm{C}_{6} \mathrm{H}_{4}-\mathrm{C}_{6} \mathrm{H}_{4}-\mathrm{SH}\right)$ (Refs. 26 and 27) observed commensurate $(\sqrt{3} \times \sqrt{3}) R 30^{\circ}$ surface lattices, but these SAMs were described as quite defective. For example, superlattice diffraction spots that are expected for a herringbone-type arrangement of the biphenyl backbones were not observed in a low-energy $\mathrm{He}$ atom diffraction experiment ${ }^{26}$ because of the small coherence length. ${ }^{30} \mathrm{We}$ suspect that the existence of two competing packing struc-

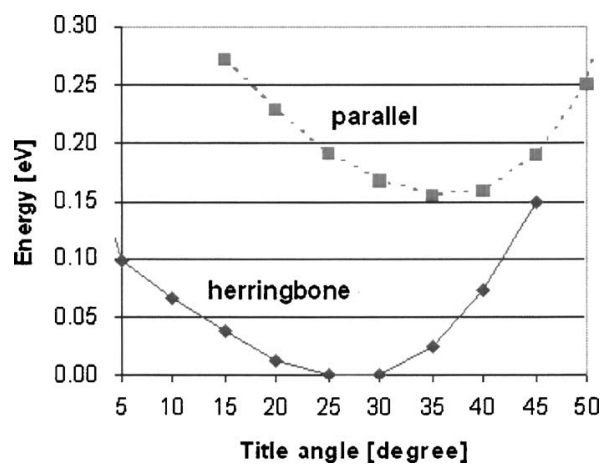

FIG. 3. Variation of the FF energy per molecule with respect to the molecular tilt angle defined via $\mathrm{S}-\mathrm{C}$ axes within the $(2 \sqrt{3} \times \sqrt{3}) R 30^{\circ}$ unit-cell herringbone (solid lines) and parallel (dashed lines) BPDT SAM packing configurations. Six layers of Au atoms have been adopted and fixed during the constrained energy minizations.

tures might contribute to such structural indeterminacy of (methyl-)mercaptobiphenyl SAMs. Indeed, in addition to the hexagonal phase of the $(\sqrt{3} \times \sqrt{3}) R 30^{\circ}$ surface lattice, a stripe phase with commensurate rectangular $(8 \times 2 \sqrt{3}) R 30^{\circ}$ surface lattice, which might be related to our less-stable parallel packing configuration, was observed. ${ }^{26}$ In a more recent scanning tunneling microscopy experiment, several other structural phases were also observed for 4-methyl$4^{\prime}$-mercaptobiphenyl SAMs. ${ }^{27}$ As will be presented shortly, we find that the BPDT SAM energy is quite insensitive to the molecule tilting. We claim that the existence of such various local minima correlates well with the experimental difficulty of making well-ordered biphenyl-based SAMs. It would be worth mentioning that SAMs based on biphenyl backbones with methyl spacers $\left[\mathrm{CH}_{3}-\mathrm{C}_{6} \mathrm{H}_{4}-\mathrm{C}_{6} \mathrm{H}_{4}-\left(\mathrm{CH}_{2}\right)_{n}-\mathrm{SH}, n\right.$ $=1-6]$ form well-ordered monolayers and additionally show an interesting even-odd effect. ${ }^{31}$ In this work, we will concentrate on the BPDT case with no methyl spacers.

In Ref. 26, a tilt angle of $19^{\circ}$ that is smaller than our results has been proposed. For comparison, we checked the variation of the BPDT SAM total energy with respect to the molecule tilting within our FFs. Taking one $(2 \sqrt{3}$ $\times \sqrt{3}) R 30^{\circ}$ unit cell of FF optimized herringbone and parallel BPDT SAMs composed of two BPDT molecules and six layers of Au electrode atoms, we performed energy minimizations while fixing all the $\mathrm{Au}$ atoms and $\mathrm{S}$ atoms and applying angle restraints on the $\mathrm{Au}-\mathrm{S}-\mathrm{C}$ bonds by $1000 \mathrm{kcal} / \mathrm{mol}=43.393 \mathrm{eV}$. The results are shown in Fig. 3. We find that the herringbone packing is more stable than the parallel packing configuration. However, within the herringbone packing configuration, we find that the energy variation with respect to the molecule tilting around the energy minimum tilt angle $\theta \approx 30^{\circ}$ is very small, smaller than $0.05 \mathrm{eV}$ per molecule in the range of $\theta \approx 15^{\circ}-35^{\circ}$.

To validate this conclusion derived from FF simulations, we performed further DFT calculations within the PerdewBurke-Ernzerhof (PBE) parameterization of the generalized gradient approximation (GGA) (Ref. 32) using the SeqQuest simulation package. ${ }^{33}$ Because the quantum-mechanical calculations are much more time consuming, we considered only three configurations: (i) herringbone $\theta \approx 15^{\circ}$, (ii) herringbone $\theta \approx 30^{\circ}$, and (iii) parallel $\theta \approx 30^{\circ}$. These three SAM 
TABLE I. Molecular tilt angles (defined via $\mathrm{S}-\mathrm{S}$ axes) and the average distances between $\mathrm{S}$ and the nearest three Au atoms in the herringbone $\theta \approx 15^{\circ}$, the herringbone $\theta \approx 30^{\circ}$, and the parallel $\theta \approx 30^{\circ} \mathrm{SAM}$ models from FF and PBE-DFT energy minimizations.

\begin{tabular}{cccc}
\hline \hline $\begin{array}{c}\text { Method (No. of } \\
\text { total/fixed Au layers) }\end{array}$ & Herringbone $\theta \approx 15^{\circ}$ & Herringbone $\theta \approx 30^{\circ}$ & Parallel $\theta \approx 30^{\circ}$ \\
\hline FF $(6 / 6)$ & $18.2^{\circ} / 2.71 \AA$ & $27.9^{\circ} / 2.71 \AA$ & $27.8^{\circ} / 2.71 \AA$ \\
FF $(6 / 1)$ & $18.4^{\circ} / 2.70 \AA$ & $26.2^{\circ} / 2.70 \AA$ & $28.0^{\circ} / 2.70 \AA$ \\
DFT $(3 / 3)$ & $13.2^{\circ} / 2.64 \AA$ & $24.5^{\circ} / 2.70 \AA$ & $27.2^{\circ} / 2.66 \AA$ \\
DFT $(3 / 1)$ & $14.0^{\circ} / 2.63 \AA$ & $24.9^{\circ} / 2.64 \AA$ & $27.5^{\circ} / 2.73 \AA$ \\
\hline \hline
\end{tabular}

configurations will be employed for the full device modeling in Sec. III, where the comparison of the first two will show the effect of molecule tilting on the $I-V$ characteristics while the comparison of the latter two will show the effect of the SAM packing.

Norm-conserving scalar-relativistic pseudopotentials ${ }^{34,35}$ were employed to remove core electrons, and orbitals were expanded in terms of a linear combination of atomic orbitals represented in the double- $\zeta$-polarization quality Gaussian functions for $\mathrm{H}, \mathrm{C}$, and $\mathrm{S}$, and the single $(5 d) /$ double$(6 s)-\zeta$-polarization Gaussian functions for $\mathrm{Au}$. The new $\mathrm{Au}$ pseudopotential and basis set have been optimized and tested for gold dimer and bulk crystal. We adopted a uniform realspace grid with 0.32-bohr spacing for the calculation of the exchange-correlation energy and potential and the Hartree potential. For the surface-parallel direction reciprocal space, $2 \times 2 \mathbf{k}_{\|}$points per $(\sqrt{3} \times \sqrt{3}) R 30^{\circ}$ cell were sampled. The total energy was minimized until the maximum forces were smaller than $0.025 \mathrm{eV} / \AA$.

Differences in the molecular geometries from FF and DFT optimizations were minimal, and some important geometrical parameters from the two calculations are compared in Table I. Energetic ordering of corresponding configurations is also summarized in Table II. Consistent with the FF results, the herringbone configuration is energetically more stable than the parallel configuration within the PBE-GGA. However, the herringbone $\theta \approx 15^{\circ} \mathrm{SAM}$ is more stable than the herringbone $\theta \approx 30^{\circ}$ SAM within the PBE-GGA. We note, however, that the energy difference between the herringbone $\theta \approx 15^{\circ}$ and $\theta \approx 30^{\circ}$ conformations is negligible: $0.003 \mathrm{eV}$ per one BPDT molecule in the case of fixing all the $\mathrm{Au}$ atoms and $0.037 \mathrm{eV}$ per one BPDT molecule and accompanying six Au atoms in the case of fixing only the bottom layer of $\mathrm{Au}$ atoms. Considering the small energy variation with the molecule tilting, the reverse placement of the energetic ordering of the herringbone $\theta \approx 15^{\circ}$ and the herring- bone $\theta \approx 30^{\circ} \mathrm{SAM}$ within our FFs is not surprising. In fact, it is well known that the GGA DFT does not describe weak van der Waals interactions accurately. ${ }^{36}$ So what we can safely conclude from the data obtained above is the small energy variation with the molecule tilting within the stable herringbone packing mode.

Summarizing, using FF and DFT calculations, we identified the ground-state herringbone packing and the lessstable parallel packing modes for the BPDT SAMs. However, the energy variation with the molecule tilting within the herringbone packing configuration was very small which leads us to conclude that the BPDT SAM is structurally not well defined. In Sec. III, we will consider the effect of the SAM packing and molecule tilting on the $I-V$ characteristics within the full BPDT SAM device geometry.

\section{DEVICE CHARACTERISTICS FROM DENSITY- FUNCTIONAL AND MATRIX GREEN'S- FUNCTION CALCULATIONS}

Molecular electronic devices we aim to model are based on SAMs with well-determined symmetric contacts through thiol bridges to metal electrodes, which can be possibly realized in crossed-wire tunnel junction ${ }^{10}$ or conducting probe atomic force microscopy ${ }^{11}$ experiments. We further assume that the device characteristics are measured at a low temperature, so that only the ground-state molecular structures need to be considered. We completed our device models by capping DFT-optimized Au-fixed SAM models obtained in Sec. II by the top $\mathrm{Au}(111)$ electrode atoms, as schematically shown in Fig. 1(b). The top Au electrode atoms were placed such that the top and bottom electrode atoms are symmetrically placed with respect to the molecules. DFT optimizations of full devices have been performed with the same calculation parameters as in the case of the one-sided SAM models (see Sec. II) by keeping $10 \AA$ or more of vacuum

TABLE II. Energetic ordering of the herringbone $\theta \approx 15^{\circ}$, the herringbone $\theta \approx 30^{\circ}$, and the parallel $\theta \approx 30^{\circ}$ SAM models at the geometries given in Table I. Units are $\mathrm{eV}$ per molecule plus accompanying Au atoms that were allowed to relax.

\begin{tabular}{cccc}
\hline \hline $\begin{array}{c}\text { Method (No. of } \\
\text { total/fixed Aulayers) }\end{array}$ & Herringbone $\theta \approx 15^{\circ}$ & Herringbone $\theta \approx 30^{\circ}$ & Parallel $\theta \approx 30^{\circ}$ \\
\hline FF $(6 / 6)$ & +0.038 & 0.000 & +0.144 \\
FF (6/1) & +0.040 & 0.000 & +0.099 \\
DFT (3/3) & -0.003 & 0.000 & +0.127 \\
DFT (3/1) & -0.037 & 0.000 & +0.146 \\
\hline \hline
\end{tabular}




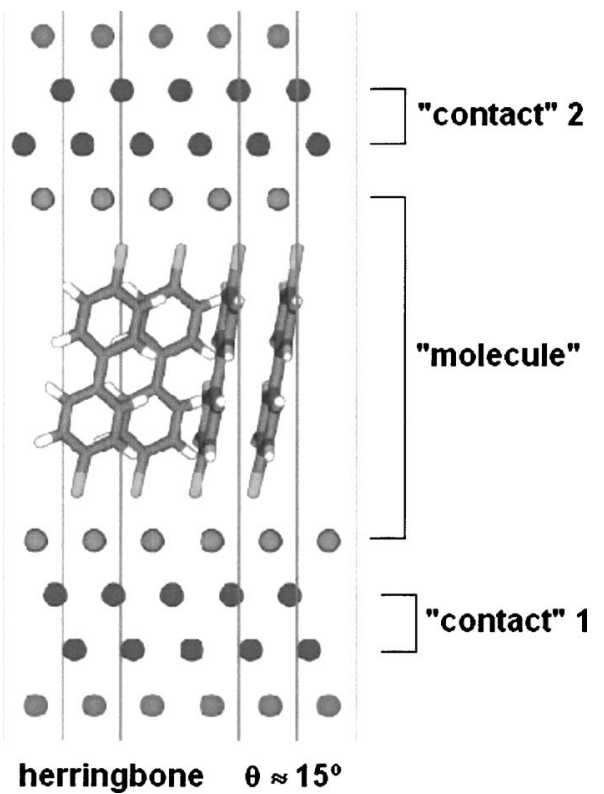

FIG. 4. Side view of $1 \times 2(2 \sqrt{3} \times \sqrt{3}) R 30^{\circ}$ cell BPDT SAM device model in the herringbone $\theta \approx 15^{\circ}$ configuration. Color codes: C (grey), $\mathrm{H}$ (white), $\mathrm{S}$ (yellow), Au included as the molecule part in the MGF calculation (orange), $\mathrm{Au}$ included as the contact part in the MGF calculation (green), and $\mathrm{Au}$ included only in the DFT calculation but excluded in the MGF calculation (sky blue).

space along the surface normal direction and allowing the top electrode to shear with respect to the bottom one. These second-stage geometry optimizations resulted in minimal changes in the position of Au atoms and molecular structures from the original one-sided SAM cases.

For the accurate calculation of the device charge transport characteristics, we adopted for the final DFT calculations four layers of $\mathrm{Au}$ electrode atoms as each electrode (Fig. 4). Along the surface-parallel direction reciprocal space, $3 \times 3 \mathbf{k}_{\|}$points per $(\sqrt{3} \times \sqrt{3}) R 30^{\circ}$ cell were sampled.

To analyze the electronic structure of the slab device models we first consider biphenyl, sulfur, and gold projections of density of states (DOS),

$$
\operatorname{DOS}(E)=\frac{1}{\Omega} \sum_{n} \int_{\tilde{\Omega}} d \mathbf{k}_{\|}\left|\phi_{n}^{\mathbf{k}_{\|}}(\mathbf{r})\right|^{2} \delta\left(E-\varepsilon_{n}^{\mathbf{k}_{\|}}\right),
$$

where $\Omega$ is the area of the reference unit cell, $\widetilde{\Omega}=(2 \pi)^{2} / \Omega$ is the area of the reciprocal unit cell, $\phi$ are DFT single-particle (Kohn-Sham) orbitals, and $\varepsilon$ are single-particle orbital energies. Projected DOS (PDOS) data of the three models calculated with energy broadening $0.05 \mathrm{eV}$ are shown in Fig. 5. Note that this is the DOS of a finite slab, which only serves as the approximation of the true semi-infinite device. DOS obtained within the semi-infinite device geometry will be presented shortly and the comparison of the two will give us an indication of the importance of taking into account the semi-infinite nature of the device. In all three cases $\mathrm{S}$ states appear about $1 \mathrm{eV}$ below $E_{F}$ followed by biphenyl states at further below. So, due to the energetic position of the $S$ states, the BPDT highest-occupied molecular-orbital (HOMO) levels are closer to the Fermi level $E_{F}$ than the lowest-unoccupied molecular-orbital (LUMO) levels. We no-

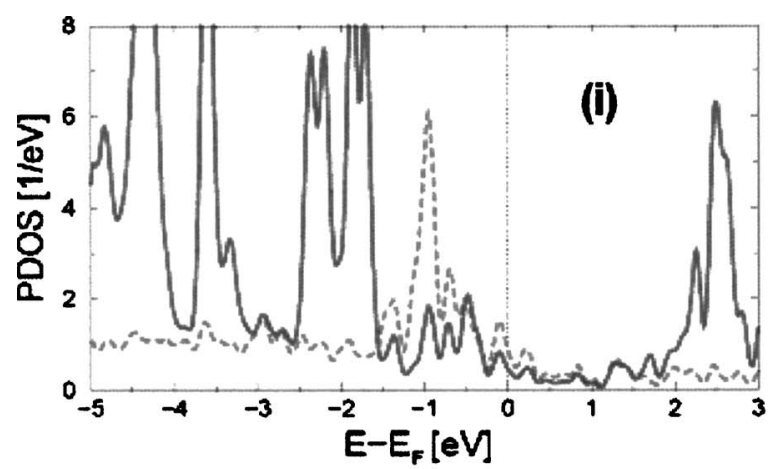

(a)

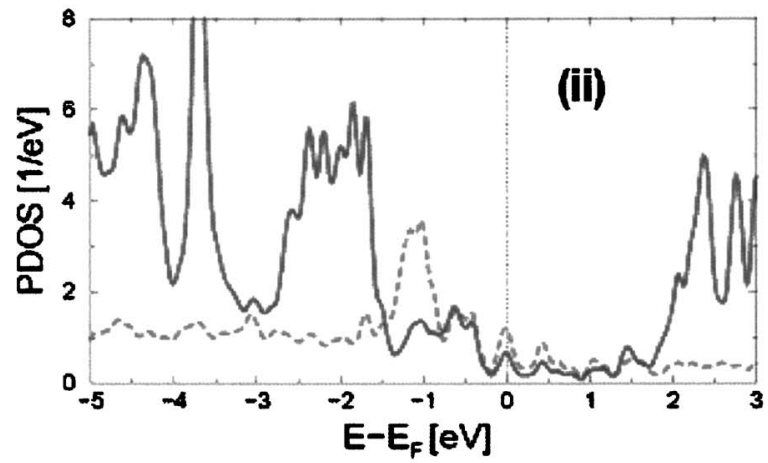

(b)

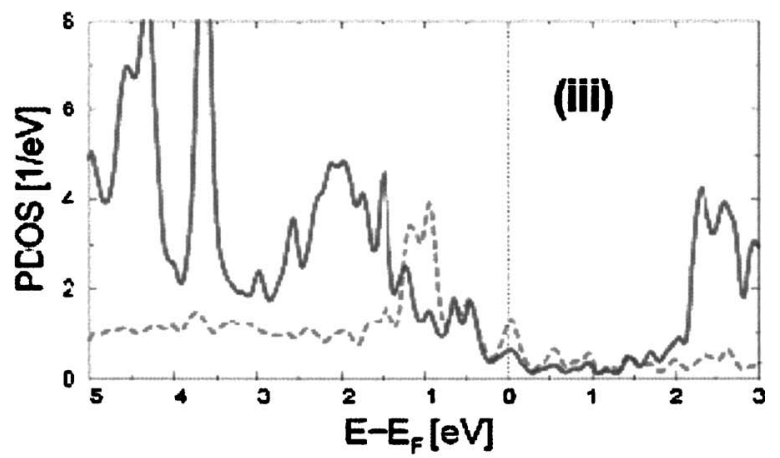

(c)

FIG. 5. DFT per molecule PDOS of BPDT SAM devices in the (a) herringbone $\theta \approx 15^{\circ}$, (b) herringbone $\theta \approx 30^{\circ}$, and (c) parallel $\theta \approx 30^{\circ}$ packing configurations. The solid lines are biphenyl PDOS and the dashed lines are $\mathrm{S}$ PDOS.

tice that the S PDOS shows very broadened peaks and that there exists finite $\mathrm{S}$ and biphenyl PDOS around $E_{F}$ or "metalinduced gap states." This indicates that thiol molecules are strongly coupled to electrodes, so localized quantum dot states will be difficult to be realized in these systems.

We next employed the MGF approach with the DFT slab calculation results as the input to consider the true semiinfinite devices. ${ }^{37,38}$ Assuming that the molecular region is set up large enough such that the coupling between two electrodes is negligible, the "molecule" part of the one-particle retarded matrix Green's function $G(E, V)=[(E+i 0+) S$ $-H(V)]^{-1}$ can be obtained for each $\mathbf{k}_{\|}$point as 


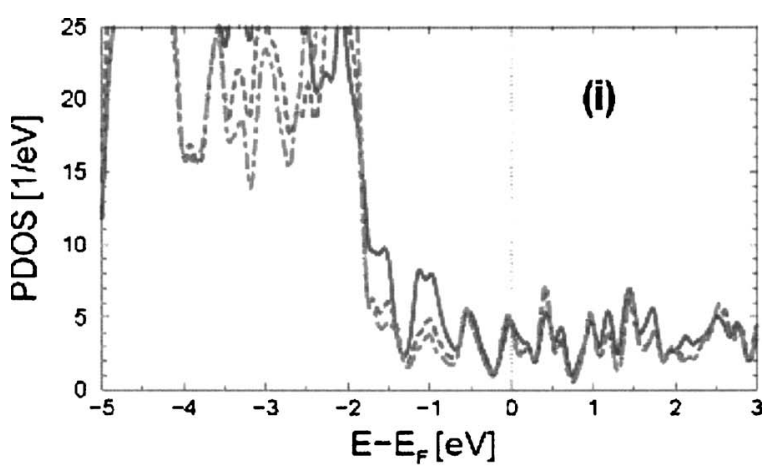

(a)

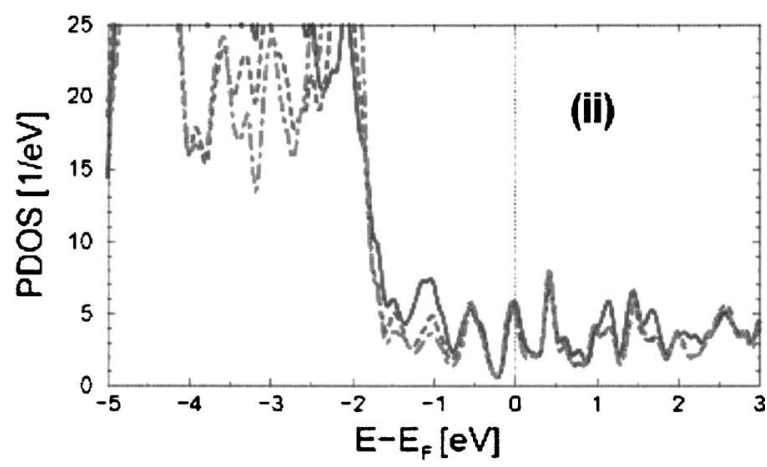

(b)

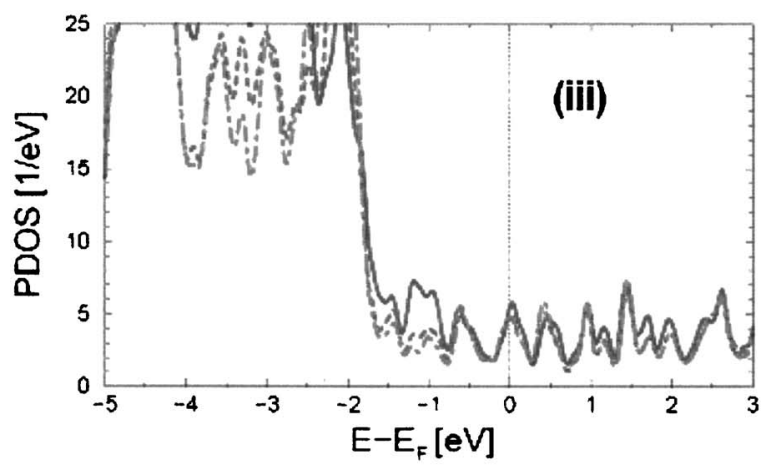

(c)

FIG. 6. DFT PDOS of Au electrode atoms in the surface (solid line), surface +1 (dashed line), and surface +2 (dot-dashed line) layers.

$$
G_{M}^{\mathbf{k}_{\|}}(E, V)=\left[E S_{M}^{\mathbf{k}_{\|}}-H_{M}^{\mathbf{k}_{\|}}(V)+\Sigma_{1}^{\mathbf{k}_{\|}}(E, V)+\sum_{2}^{\mathbf{k}_{\|}}(E, V)\right]^{-1},
$$

where $\Sigma_{a}^{\mathbf{k}_{\|}}$is the self-energy,

$$
\sum_{a}^{\mathbf{k}_{\|}}(E)=x_{a}^{\mathbf{k}_{\|}}(E) g_{s}^{\mathbf{k}_{\|}}(E) x_{a}^{\mathbf{k}_{\|}^{+}}(E) \quad(a=1,2),
$$

where $g_{S}^{\mathbf{k}_{\|}}$is the surface Green's function and $x_{a}^{\mathbf{k}_{\|}}$is the offdiagonal "molecule"-“contact" coupling part of $E S_{a}^{\mathbf{k}_{\|}}-H_{a}^{\mathbf{k}_{\|}}$. The self-energy is a non-Hermitian quantity that includes the information on the shift of molecular energy levels (Hermitian part) and the broadening (anti-Hermitian part) due to the coupling of molecules to electrodes.

To properly determine the "molecule" part, we consider the DFT PDOS of Au electrode atoms shown in Fig. 6. For the energy range of our interest, $E_{F}-2 \sim E_{F}+2 \mathrm{eV}$, we observe that while the PDOS of the surface +1 and surface +2 layers are essentially identical, the surface layer PDOS deviates from those of deeper layers. We thus define the BPDT molecules plus one layer of Au electrode atoms as the "molecule" part. As the "contact" region, we adopted the surface +1 and surface +2 layers of Au electrode atoms. This calculation setup is shown in Fig. 4.

To obtain the semi-infinite surface Green's function $g_{S}^{\mathbf{k}_{\|}}$, we performed two separate bulk metal phase DFT calculations with the isolated slabs as the unit cells with the $\mathbf{k}_{\|}$ sampling equivalent to that of the main device calculation and iteratively extracted $g_{S}^{\mathbf{k}_{\|}}$. ${ }^{13,39}$ Two $\mathbf{k}_{\perp}$ points have been sampled along the surface normal direction, and the Hamiltonian and overlap matrices have been Fourier transformed to the real space along the surface-normal direction for the calculation of $g_{S} \mathbf{k}_{S}$. For devices made of small molecules considered in this work, the size of electrodes is larger than the molecule itself. So, the computation of $g_{S}^{\mathbf{k}_{\|}}$represents the most time-consuming step in our $I-V$ calculations. We finally aligned the energy levels of $x_{a}^{\mathbf{k}_{\|}}$and $g_{S}^{\mathbf{k}_{\|}}$by matching the Fermi levels from the three DFT calculations, and constructed $\Sigma_{a}^{\mathbf{k}_{\|}}$for each sampling energy point $E$. Because our MGF method is based on periodic DFT calculations of devices and electrodes, we can treat the SAM molecules and semi-infinite electrodes more naturally and accurately compared with other implementations based on zero-dimensional quantum chemistry codes.

We first consider the molecular projection of DOS at the zero bias,

$$
\operatorname{DOS}(E)=\frac{1}{2 \pi \Omega} \int_{\tilde{\Omega}} d \mathbf{k}_{\|} \operatorname{Tr}\left[A_{M}^{\mathbf{k}_{\|}}(E) S_{M}^{\mathbf{k}_{\|}}\right]
$$

where $A=i\left(G-G^{+}\right)$is the spectral function, shown in Fig. 7. Note that this is the PDOS of truly semi-infinite devices while the PDOS of Fig. 5 is that of a finite slab, so the difference between the two represents the effect of coupling of molecules to semi-infinite electrodes. Also, note that while we needed to include an artificial energy-level broadening in Eq. (1), energy-level broadening is now described in an $a b$ initio fashion by the anti-Hermitian part of self-energies [Eq. (3)].

Comparing DFT PDOS of Fig. 5 and MGF PDOS of Fig. 7, we find, e.g., that the true device level broadening (Fig. 7) is smaller than the $0.05 \mathrm{eV}$ used in the DFT PDOS calculation (Fig. 5). The most important difference for our purpose is, however, the character of the metal-induced gap states near $E_{F}$ : we observe much smoother curves in the MGF data (Fig. 7) than in the DFT data (Fig. 5). This difference can be easily understood in that the continuous metalinduced gap spectrum in the semi-infinite device geometry (Fig. 7) should be approximated by discrete states within the finite slab model (Fig. 5). Metal-induced gap states as well as the relative position of broadened molecular orbitals with respect to $E_{F}$ are determining factors for the transmission characteristics of our BPDT SAM devices. We emphasize again that these can be unambiguously determined only when we properly model the bulk nature of electrodes and 


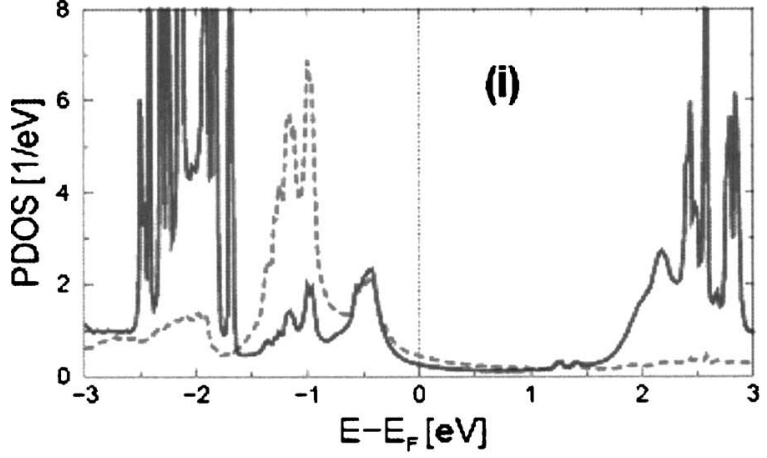

(a)

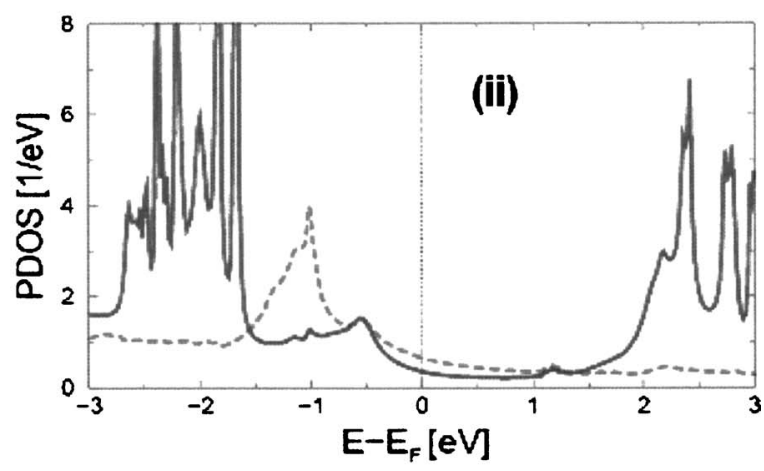

(b)

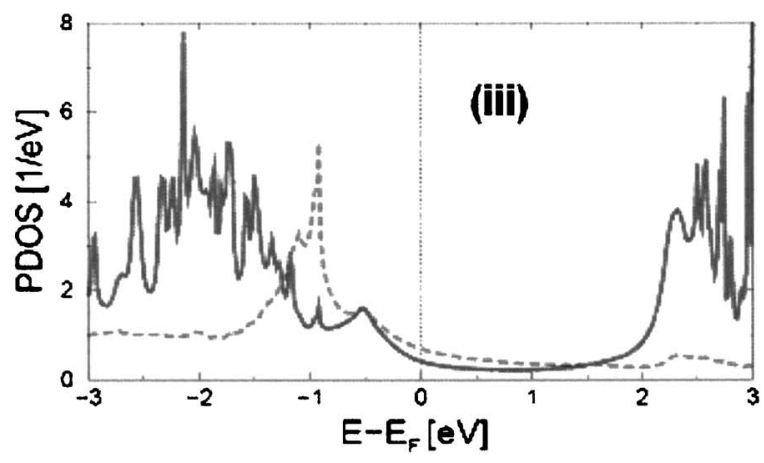

(c)

FIG. 7. MGF per molecule PDOS of BPDT SAM devices in the (a) herringbone $\theta \approx 15^{\circ}$, (b) herringbone $\theta \approx 30^{\circ}$, and (c) parallel $\theta \approx 30^{\circ}$ packing configurations. The solid lines are biphenyl PDOS and the dashed lines are S PDOS.

the coupling of molecules with electrodes in a proper manner by incorporating a large number of metal electrode atoms.

Focusing now on the comparison of the PDOS of the three device models in Fig. 7, we first find that, for the $S$ PDOS located around $E_{F}-1.0 \mathrm{eV}$, model (i) shows a much sharper peak than models (ii) and (iii). This indicates that the herringbone packing with $\theta \approx 15^{\circ}$ provides the stronger localization of the $S$ level than the herringbone and parallel $\theta \approx 30^{\circ}$ models. Second, for the phenyl contributions distributed in the energy range of $E_{F}-3.0 \mathrm{eV}-E_{F}-1.5 \mathrm{eV}$, we observe that model (iii) shows a much lower and broader peak than models (i) and (ii), due to the stronger phenyl band formation.

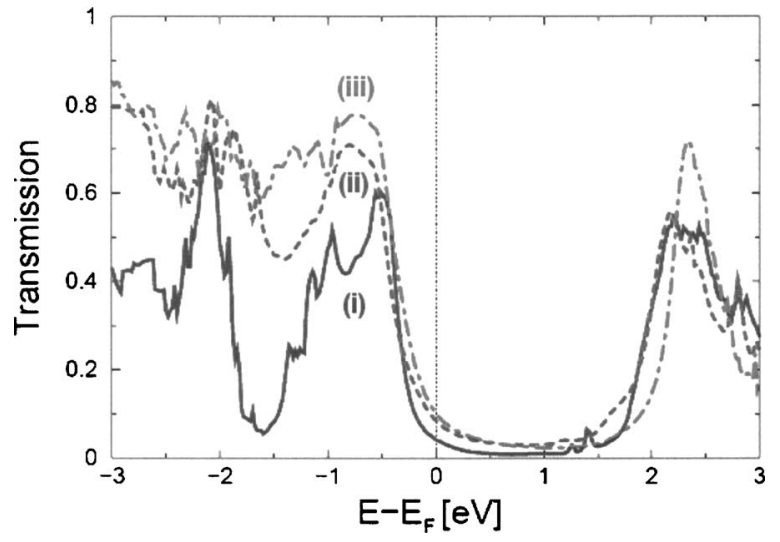

FIG. 8. Transmission of BPDT SAM devices with herringbone $\theta \approx 15^{\circ}$ (solid line), (b) herringbone $\theta \approx 30^{\circ}$ (dashed line), and (c) parallel $\theta \approx 30^{\circ}$ (dot-dashed line) packing configurations.

We next consider the device $I-V$ characteristics computed by invoking the Landauer-Büttiker formalism, ${ }^{38}$ which relates the linear-response conductance to the transmission probability $T$ as

$$
I(V)=\frac{2 e}{h} \int_{\mu_{1}}^{\mu_{2}} d E T(E, V)\left[f\left(E-\mu_{1}\right)-f\left(E-\mu_{2}\right)\right],
$$

where $\mu_{1}$ and $\mu_{2}$ are the chemical potentials of electrodes 1 and 2 , the bias voltage $V$ is symmetrically distributed, $\mu_{1}$ $=E_{F}-0.5 \mathrm{eV}$ and $\mu_{2}=E_{F}+0.5 \mathrm{eV},{ }^{12}$ and $f$ is the Fermi-Dirac distribution function. We assume the zero-temperature conductance and neglect noncoherent charge transport processes that could couple different transverse modes. So, the calculation of $I-V$ characteristics amounts to the evaluation of the transmission function through independent $\mathbf{k}_{\|}$channels and their summation over the surface Brillouin zone,

$$
T(E, V)=\frac{1}{\Omega} \int_{\tilde{\Omega}} d \mathbf{k}_{\|} T^{\mathbf{k}_{\|}}(E, V) .
$$

The transmission function at $\mathbf{k}_{\|}, T^{\mathbf{k}_{\|}}(E)$, are expressed in terms of Green's functions as ${ }^{12,13}$

$$
T^{\mathbf{k}_{\|}}(E, V)=\operatorname{Tr}\left[\Gamma_{1}^{\mathbf{k}_{\|}}(E, V) G_{M}^{\mathbf{k}_{\|}}(E, V) \Gamma_{2}^{\mathbf{k}_{\|}}(E, V) G_{M}^{\mathbf{k}_{1}^{+}}(E, V)\right]
$$

where

$$
\Gamma_{a}^{\mathbf{k}_{\|}}(E, V)=i\left[\Sigma_{a}^{\mathbf{k}_{\|}}(E, V)-\Sigma_{a}^{\mathbf{k}_{\|}^{+}}(E, V)\right] \quad(a=1,2)
$$

describes the broadening of molecular energy levels (inverse lifetime) due to the coupling to electrodes. Details of our implementation will be reported elsewhere.

In calculating the $I-V$ characteristics, we adopted the approximation of employing the zero-bias transmission $T(E, V=0)$ to compute the current $I$ at a finite bias $V$, $T(E, V) \approx T(E, V=0) \equiv T(E)$. Finite- $V$ transmission $T(E, V)$ should be close to $T(E)$ in the low- $V$ nonresonant transmission regime, ${ }^{16}$ but large- $V$ region $(|V|>0.5 \mathrm{~V}) I-V$ curves in this report should be taken as qualitative.

The zero-bias transmission functions of the three device models are shown in Fig. 8, and the corresponding $I-V$ curves [obtained within the approximation of $T(E, V)$ $\approx T(E, V=0)]$ are shown in Fig. 9. In the low-bias voltage 


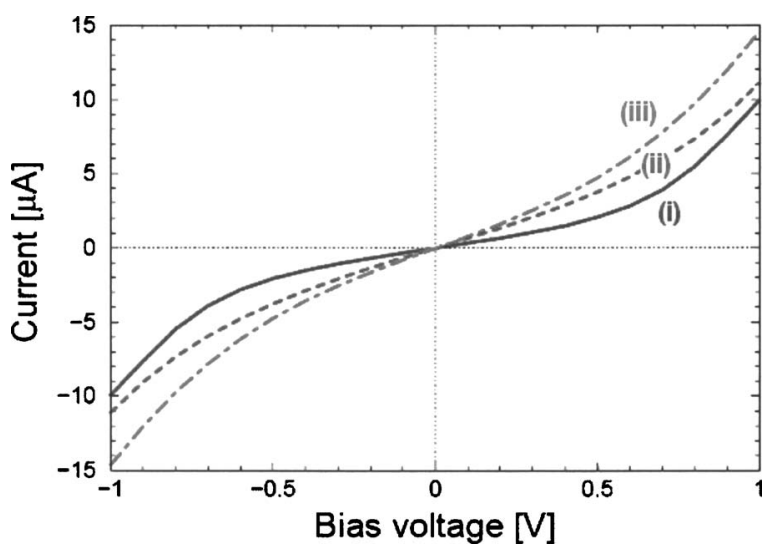

FIG. 9. Per molecule $I-V$ characteristics of BPDT SAM devices with herringbone $\theta \approx 15^{\circ}$ (solids line), herringbone $\theta \approx 30^{\circ}$ (dashed line), parallel $\theta \approx 30^{\circ}$ (dot-dashed line) packing configurations.

region $(|V| \lesssim 0.2 \mathrm{~V})$, we observe that the $I-V$ characteristics of the herringbone and parallel SAMs with the molecular tilt angle $\theta \approx 30^{\circ}$ [models (ii) and (iii)] are quite similar, while the current through the herringbone $\theta \approx 15^{\circ} \mathrm{SAM}$ [model (i)] is smaller than that in the other two. This results from the previously discussed stronger delocalization of $S$ levels around $E_{F}-1.0 \mathrm{eV}$ and the correspondingly bigger S PDOS tails at $E_{F}$ with the molecule tilting (Fig. 7). The difference in the $I-V$ characteristics of models (ii) and (iii) in the lowbias voltage region is small, because the phenyl states located in the energy range further away from $E_{F}$ (below $E_{F}$ $-1.5 \mathrm{eV}$ ) have smaller tails around $E_{F}$ and accordingly the difference in their transmission characteristics (Fig. 8) is negligible.

In the high-bias voltage region $(|V| \geq 0.5 \mathrm{~V})$, we observe that the $I-V$ characteristics of the three models diverge from each other. In particular, with the increasing bias, the current of model (ii) increases faster than that of model (iii) (Fig. 9). This is due to the stronger phenyl band formation within the parallel SAM packing configuration (Fig. 7). Moreover, we would expect that even bigger differences in the $I-V$ characteristics of the three models can be observed in the higherbias voltage region: Assuming that the zero-bias transmission functions (Fig. 8) do not change with the bias, their bigger differences in the energy range below $E_{F}-0.5 \mathrm{eV}$ will result in bigger differences in their $I-V$ characteristics at $|V|$ $\gtrsim 1 \mathrm{~V}$ (Fig. 9). So, we conclude that in the high-bias voltage region both intramolecular conformation (molecule tilting) and intermolecular interaction (phenyl band formation) can affect the SAM $I-V$ characteristics.

\section{CONCLUSIONS}

In this work, we studied BPDT SAM molecular wires at the $(\sqrt{3} \times \sqrt{3}) R 30^{\circ}$ packing density sandwiched between $\mathrm{Au}(111)$ electrodes, with emphasis on the conformation- $I-V$ characteristics correlation. Using large-scale FF and DFT calculations of BPDT SAMs, we first identified two competing herringbone and parallel packing modes, and found that the herringbone packing configuration is energetically more favorable than the parallel packing by $\sim 0.15 \mathrm{eV}$ per molecule. Within the more-stable herringbone packing configu- ration, we found, however, that the potential-energy surface as a function of the molecular tilt angle $\theta$ is very flat, with $\lesssim 0.04 \mathrm{eV}$ per molecule difference between $\theta \approx 15^{\circ}$ and $\theta$ $\approx 35^{\circ}$. This theoretical finding of the energetic indeterminacy correlates well with the experimental observation of the difficulty in making well-ordered biphenylthiol-based SAMs on the $\mathrm{Au}(111)$ surface. Next, we prepared three device models based on three BPDT SAMs: (i) herringbone SAM with $\theta$ $\approx 15^{\circ}$, (ii) herringbone SAM with $\theta \approx 30^{\circ}$, and (iii) parallel SAM with $\theta \approx 15^{\circ}$, and computed their $I-V$ characteristics employing the MGF method and the Landauer-Büttiker formula. We found that for the low-bias voltage region $(|V|$ $\lesssim 0.2 \mathrm{~V}$ ) the $I-V$ characteristics of models (ii) and (iii) are quite similar and their conductance is larger than that of model (i). From this, we concluded that the intramolecular conformation with respect to electrodes (localization/ delocalization character of the sulfur states) is the principal factor that determines the low- $V$ BPDT SAM $I-V$ characteristics. On the other hand, for the increased bias $(|V|$ $\gtrsim 0.5 \mathrm{~V}$ ), we observed that the conductance of the three device models diverge from each other due to the electrodesurface-direction intermolecular interactions (phenyl band formation). Thus, at the (ideal) very dense SAM packing condition, we expect that both the intramolecular conformation and intermolecular interaction would affect the high-bias BPDT SAM $I-V$ characteristics. Because the molecular tilt angle and packing modes are not well characterized while its $I-V$ characteristics depend sensitively on such conformational details, we consider the BPDT SAM as a poor device candidate. In any case, we propose that such variations in the structures and $I-V$ characteristics should be taken into account when one adopts BPDT SAMs as a device component. This shows a promising possibility of applying computational tools to characterize the performance of molecular electronics device candidates prior to more expensive and difficult experimental investigations.

\section{ACKNOWLEDGMENTS}

We thank Dr. Yun Hee Jang and Dr. Wei-Qiao Deng for helpful discussions. The computational work was initiated with support by the National Science Foundation (NSF) Nanotechnology and Interdisciplinary Research Initiative (NIRT) and supported by the Microelectronics Advanced Research Corporation (MARCO) and its Focus Centers on Functional Engineered NanoArchitectonics (FENA). The facilities of the MSC were supported by ONR-DURIP, ARODURIP, NSF-MRI, and the Beckman Institute. One of the authors (Y.-H. K.) also acknowledges the support from IPAM at UCLA and KIAS.

${ }^{1}$ C. Joachim, J. K. Gimzewski, and A. Aviram, Nature (London) 408, 541 (2000).

${ }^{2}$ J. R. Heath and M. A. Ratner, Phys. Today 56(5), 43 (2003).

${ }^{3}$ A. Nitzan and M. A. Ratner, Science 300, 1384 (2003).

${ }^{4}$ J. M. Tour, L. Jones II, D. L. Pearson et al., J. Am. Chem. Soc. 117, 9529 (1995).

${ }^{5}$ M. A. Reed, C. Zhou, C. J. Muller, T. P. Burgin, and J. M. Tour, Science 278, 252 (1997); J. Chen, M. A. Reed, A. M. Rawlett, and J. M. Tour, Science 286, 1550 (1999).

${ }^{6}$ T. Ishida, W. Mizutani, N. Choi, U. Akiba, M. Fujihira, and H. Tokumoto, J. Phys. Chem. B 104, 11680 (2000); T. Ishida, W. Mizutani, Y. Aya, H. 
Ogiso, S. Sasaki, and H. Tokumoto, J. Phys. Chem. B 106, 5886 (2002)

${ }^{7}$ Z. J. Donhauser, B. A. Mantooth, K. F. Kelly et al., Science 292, 2303 (2001)

${ }^{8}$ S. Hong, R. Reifenberger, W. Tian, S. Datta, J. Henderson, and C. P. Kubiak, Superlattices Microstruct. 28, 289 (2000).

${ }^{9}$ J. Reichert, R. Ochs, D. Beckmann, H. B. Weber, M. Mayor, and H. V. Löhneysen, Phys. Rev. Lett. 88, 176804 (2002).

${ }^{10}$ J. G. Kushmerick, D. B. Holt, J. Naciri, M. H. Moore, and R. Shashidhar, Phys. Rev. Lett. 89, 086802 (2002); J. G. Kushmerick, J. G. Holt, S. K Pollack et al., J. Am. Chem. Soc. 124, 100654 (2002)

${ }^{11}$ D. J. Wold, R. Haag, M. A. Rampi, and C. D. Frisbie, J. Phys. Chem. B 106, 2813 (2002)

${ }^{12}$ S. Datta, W. Tian, S. Hong, R. Reifenberger, J. Henderson, and C. P. Kubiak, Phys. Rev. Lett. 79, 2530 (1997).

${ }^{13}$ P. Damle, A. W. Ghosh, and S. Datta, Phys. Rev. B 64, 201403 (2001); P. Damle, A. W. Ghosh, and S. Datta, Chem. Phys. 281, 171 (2002).

${ }^{14}$ M. D. Ventra, S. T. Pantelides, and N. D. Lang, Phys. Rev. Lett. 84, 979 (2000).

${ }^{15}$ E. G. Emberly and G. Kirczenow, Phys. Rev. B 64, 235412 (2001).

${ }^{16}$ Y. Xue, S. Datta, and M. A. Ratner, J. Chem. Phys. 115, 4292 (2001); Y. Xue and M. A. Ratner, Phys. Rev. B 68, 115406 (2003).

${ }^{17}$ P. A. Derosa and J. M. Seminario, J. Phys. Chem. B 105, 471 (2001).

${ }^{18}$ J. Heurich, J. C. Cuevas, W. Wenzel, and G. Schön, Phys. Rev. Lett. 88, 256803 (2002).

${ }^{19}$ J. Taylor, M. Brandbyge, and K. Stokbro, Phys. Rev. Lett. 89, 138301 (2002); J. Taylor, M. Brandbyge, and K. Stokbro, Phys. Rev. B 68 121101(R) (2003); K. Stokbro, J. Taylor, M. Brandbyge, J.-L. Mozos, and P. Ordejón, Comput. Mater. Sci. 27, 151 (2003).

${ }^{20}$ C.-C. Kaun, B. Larade, and H. Guo, Phys. Rev. B 67, 121411(R) (2003).

${ }^{21}$ Y.-H. Kim, S. S. Jang, Y. H. Jang, and W. A. Goddard III, Phys. Rev. Lett. 94, 156801 (2005).

${ }^{22}$ S. L. Mayo, B. D. Olafson, and W. A. Goddard, J. Phys. Chem. 94, 8897 (1990)
${ }^{23}$ S. S. Jang, Y. H. Jang, Y.-H. Kim et al., J. Am. Chem. Soc. 127, 1563 (2005).

${ }^{24}$ D. J. Lavrich, S. M. Wetterer, S. L. Bernasek, and G. Scoles, J. Phys. Chem. B 102, 3456 (1998).

${ }^{25}$ A. K. Rappé and W. A. Goddard III, J. Phys. Chem. 95, 3358 (1991).

${ }^{26}$ T. Y. B. Leung, P. Schwartz, G. Scoles, F. Schreiber, and A. Ulman, Surf. Sci. 458, 34 (2000).

${ }^{27}$ W. Azzam, C. Fuxen, A. Birkner, H.-T. Rong, M. Buck, and C. Wöll, Langmuir 19, 4958 (2003).

${ }^{28}$ D. Frenkel and B. Smit, Understanding Molecular Simulation, 2nd ed. (Academic, San Diego, CA, 2001).

${ }^{29}$ E. Sabatani, J. Cohen-Boulakia, M. Bruening, and I. Rubinstein, Langmuir 9, 2974 (1993); Y.-T. Tao, C.-C. Wu, J.-Y. Eu, and W.-L. Lin, ibid. 13, 4018 (1997).

${ }^{30}$ C. Fuxen, W. Azzam, R. Arnold, G. Witte, A. Terfort, and C. Wöll, Langmuir 17, 3689 (2001).

${ }^{31}$ W. Azzam, P. Cyganik, G. Witte, M. Buck, and D. J. Wold, Langmuir 19, 8262 (2003); P. Cyganik, M. Buck, W. Azzam, and D. J. Wold, J. Phys. Chem. B 108, 4989 (2004); P. Cyganik and M. Buck, J. Am. Chem. Soc. 126, 5960 (2004)

${ }^{32}$ J. P. Perdew, K. Burke, and M. Ernzerhof, Phys. Rev. Lett. 77, 3865 (1996).

${ }^{33}$ P. Schultz, SeqQuest code project, Sandia National Laboratories (http:// www.cs.sandia.gov/ paschul/Quest/).

${ }^{34}$ D. R. Hamann, Phys. Rev. B 40, 2980 (1989).

${ }^{35}$ N. Troullier and J. L. Martins, Phys. Rev. B 43, 8861 (1991).

${ }^{36}$ W. Koch and M. C. Holthausen, A Chemist's Guide to Density Functional Theory, 2nd ed. (Wiley, Weinheim, 2001).

${ }^{37}$ A. R. Williams, P. Feibelman, and N. D. Lang, Phys. Rev. B 26, 5433 (1982).

${ }^{38}$ S. Datta, Electronic Transport in Mesoscopic Systems (Cambridge University Press, Cambridge, UK, 1995).

${ }^{39}$ D. H. Lee and J. D. Joannopoulos, Phys. Rev. B 23, 4997 (1981). 